\title{
Teorización sistémica de las garantías de los derechos fundamentales en Ecuador
}

\section{Theorization of a guarantee system of fundamental rights in Ecuador}

\author{
Paúl Hernán Mancero Carrillo. ${ }^{1}$
}

\begin{abstract}
.
DOI: https://doi.org/10.33262/cienciadigital.v3i3.2.1.815

Behind some cold constitutional norms in Ecuador, it is feasible and appropriate to carry out a theorization about the establishment of a system of guarantees in our legal system, in terms of the protection and guarantee of human rights

That is, far away are the mechanisms contemplated in our Constitutional Charter for the protection of rights, whether they are isolated, not systematic and circumstantial. Quite contrary to the common belief, the constitutional articles establish a series of mechanisms for the effective implementation of them, often without suspecting that we can raise a theory about this reality and use it in the daily life of the citizens as well as a contribution academic to understand this reality. In the present project we have mainly used bibliographic references and works of national and international authors with high academic authority. The final result consists of discovering the existence and theorization of a system of guarantees of rights that is underlying a constitutional articulation, and this compared with doctrinal and theoretical points. Finally, as a conclusion, it can be pointed out that our country has an extensive system of rights guarantees, because several principles, legal rules and constitutional institutions are interconnected.
\end{abstract}

Keywords: Theorization, system, guarantee, fundamental rights, constitution

\section{Resumen}

Detrás de unas frías normas constitucionales en el Ecuador, es factible y adecuado explorar una teorización sobre el establecimiento de un sistema de garantías en

\footnotetext{
${ }^{1}$ Escuela Superior Politécnica de Chimborazo, Facultad de Administración de Empresas. Riobamba, Ecuador paul.mancero@espoch.edu.ec
} 
nuestro ordenamiento jurídico, en cuanto a la protección y garantía de los derechos fundamentales. Es decir, lejos está de qué mecanismos contemplados en nuestra Carta Constitucional para la tutela de los derechos, sean aislados, no sistemáticos y coyunturales. Muy al contrario de lo que se cree, el articulado fundamental establece una serie de mecanismos para la implementación efectiva de los mismos, muchas veces sin sospechar que podemos levantar una teoría sobre esta realidad y utilizarla tanto en la vida diaria de la ciudadanía; así como un aporte académico para entender esta realidad. En el presente trabajo, hemos utilizado de manera principal, las referencias bibliográficas y obras de autores nacionales e internacionales con alta autoridad académica. El resultado final consiste en descubrir la existencia y teorización de un sistema de garantías de los derechos que se encuentra subyacente en el articulado constitucional, esto comparado con los señalamientos doctrinarios y teóricos. Finalmente como conclusión se puede señalar que en Ecuador se puede teorizar en un amplio sistema de garantías de los derechos fundamentales, en virtud de que están interconectados principios, reglas jurídicas e instituciones constitucionales, aunque esta factibilidad ha pasado ignorada o desapercibida.

Palabras claves: Teorización, sistema, garantía, derechos, fundamentales, constitución

\section{Introducción}

El presente trabajo en el marco de la declaración teórica de un Estado Constitucional de Derechos y Justicia, aborda los elementos fundamentales expresados en la Constitución de la República del Ecuador, en cuanto a la garantía de los derechos fundamentales para ecuatorianos y extranjero que residentes en el país, pero a diferencia de otros trabajos eminentemente segmentados y aislados por cada tipo de mecanismo de tutela, lo que hace es un esfuerzo por encontrar toda una teorización para encontrar una sistematización conceptual, doctrinaria y normativa en la protección de derechos fundamentales, la misma que subyace en el contenido y que es menester evidenciarlo para lograr una compresión integral, así como la aplicación efectiva y eficaz del sistema de garantías propuesto en el presente trabajo.

\section{Metodología}

Hablar de garantías constitucionales no tiene sentido si no se habla de Derechos. Los Derechos son concebidos, desde los comienzos de la modernidad, como aquellas facultades o poderes subjetivos que se constituyen en los límites básicos al poder y a la acción del Estado. En efecto, los derechos, desde su primera acepción, que proviene del primer contractualismo medieval, (Padua, 2001) bien en la visión de los contractualistas clásicos, (Hobbes, 1980), (Locke, 2002), (Rousseau, 1983) son concebidos como límites a la acción y al poder estatal.

En ese sentido, el constitucionalismo no sería lo que es sin los derechos. De acuerdo con el Artículo 16 de la Declaración de los Derechos del Hombre y del Ciudadano, que estipula 
"Toda comunidad en la que no esté establecida la separación de poderes ni garantizados los derechos carece de Constitución"; mientras que las democracias Constitucionales contemporáneas se fundamentan en la consideración de que la garantía efectiva de los derechos y las libertades es la finalidad primera y primordial de la organización estatal.

Para cumplir con esta finalidad los Estados Constitucionales tienen establecidos institucionalmente una serie de mecanismos jurídicos o instrumentos reforzados de protección que permiten o hacen posible evitar, mitigar o reparar la vulneración de un derecho establecido en la Constitución, que se conocen como garantías. A tal punto son importantes estas garantías en el mundo jurídico contemporáneo que son consideradas por la mayoría de los autores como el rasgo que diferencia al Estado Constitucional de los anteriores modelos de Estado y en tal virtud, sin ellas los derechos no serían más declaraciones retóricas.(Guastini, 2009)

Pero históricamente no siempre fue así: en los Estados de legalidad, (las versiones del Estado de Derecho que rigieron en el siglo XIX), solo los derechos individuales tenían reconocimiento constitucional pero carecían de garantías; En los Estados Sociales, por su parte, especialmente de los países centrales del capitalismo, como consecuencia del pacto Capital - Trabajo se amplía enormemente el catálogo de derechos con la incorporación, en todas las constituciones de la segunda post guerra, de un amplio listado de derechos económicos y sociales, (Pérez Luño, 1998) sin embargo su garantía jurídica era totalmente insuficiente porque en el mejor de los casos la teoría tradicional de los derechos son consideradas garantías institucionales que tan solo definen deberes de actuación de los poderes públicos en la esfera socioeconómica, pero que como su nombre indica, no reconocen directamente pretensiones o facultades subjetivas a los ciudadanos (Pérez Luño, 1998)); En los Estados sociales de mercado, aquellos aparecidos en el mundo entero a raíz de la "revolución conservadora" de los años 80 del siglo pasado, estas condiciones de desjuridificación de los derechos sociales se agudiza a tal punto que las débiles garantías jurídicas de los derechos, desaparecen paulatinamente al ritmo de la retracción del Estado; y solo se recuperan en un sentido fuerte con la consolidación de lo que se ha dado en denominar los nuevos Estados Constitucionales que han aparecido en el mundo entero y especialmente en Latinoamérica en los últimos 15 años donde TODOS los derechos, sin importar su origen, o ámbito de protección, están garantizados a través de distintos tipos de garantía constitucional.

Aparte de la garantía política general que representa la propia existencia del Estado de Derecho, La teoría de los derechos reconoce distintos tipos o niveles de tutela jurídica para los Derechos: En primer lugar están las garantías normativas, en segundo término están las garantías institucionales y por último, se reconocen también las garantías jurisdiccionales.

En el caso ecuatoriano como veremos este conjunto de garantías se ve complementada por un tipo adicional de garantías relativas a las políticas públicas que se encuentran reconocidas en el art. 85 de la constitución aprobada por el pueblo ecuatoriano el 20 de octubre de 2008.

Las garantías normativas son aquellos principios y reglas encaminadas a conseguir que los derechos fundamentales estén efectivamente asegurados como las normas que son, se limiten al mínimo sus restricciones, y se asegure su adecuado resarcimiento cuando se han 
producido daños como consecuencia de su vulneración por parte de los poderes públicos o sus agentes. La principal garantía normativa es el conocido principio general de Supremacía de la Constitución en virtud del cual la Carta Fundamental es la norma que prevalece sobre cualquier otra y por tanto los ciudadanos y los poderes están sujetos al texto constitucional y al resto del ordenamiento. En el caso ecuatoriano esta garantía normativa esta expresada de forma diferente en distintos enunciados normativos entre los que sobresalen el artículo 424 de la Constitución de la República

Así mismo existen otras garantías normativas como el deber de respeto a los derechos establecido en el Artículo 11 numeral 9, según el cual "el más alto deber del Estado consiste en respetar y hacer respetar los derechos garantizados en la Constitución”.

Esta garantía normativa general se ve complementada por otro mecanismo de idéntica naturaleza: la rigidez e inalterabilidad constitucional, destinado a evitar la alteración del contenido e identidad de la propia constitución. En el caso de los derechos esta garantía normativa se expresa en el procedimiento de reforma especialmente rígido del catálogo de derechos y en la prohibición de restricción del contenido de los derechos, establecida en el numeral 4 del artículo 11 de la Constitución,

Otra garantía normativa importante es la obligación reparatoria definida en el inciso 2 del citado numeral que dispone la obligación del Estado de reparar las violaciones de los derechos derivada de las acciones u omisiones de sus agentes en ejercicio de sus cargos, o por la falta o deficiente prestación de servicios públicos.

Finalmente con tal nombre, (garantías normativas), los constituyentes de Montecristi establecieron un novedoso mecanismo, inédito en el país, que asegura la sujeción de la Asamblea Nacional, y de cualquier otro órgano que tenga potestad normativa reconocida por la Constitución, a los derechos establecidos en la Constitución y en los tratados internacionales de derechos humanos, con lo que todo acto normativo está limitado por el contenido y eficacia de los derechos fundamentales.

Las garantías institucionales o extrajudiciales ${ }^{i}$ son los mecanismos de Protección que la Constitución otorga no ya a los derechos fundamentales de las personas, sino a determinadas organizaciones o instituciones valiosas desde el punto de vista del constituyente, a las que asegura un núcleo o reducto indisponible para el legislador.

Ejemplos de este tipo de garantías son el principio de separación de poderes, el reconocimiento del carácter laico del Estado, también conocido como separación entre iglesia y el Estado, el principio de legalidad, la existencia de un órgano independiente ya autónomo (Corte o Tribunal Constitucional) que garantice la supremacía de la Constitución, y la labor del defensor del pueblo, entre otros.

En cuanto al principio de separación de poderes se conoce en la doctrina como la segunda columna vertebral del Estado Constitucional (Villa Casado, 2007) y consiste en una forma de organización del poder soberano del Estado que mediante su separación en distintas funciones evita su acumulación en una sola persona o institución 
Como se sabe la primera versión de la teoría de la separación de funciones del Estado la encontramos en la obra de John Locke, pero fue Montesquieu el que en el siglo XVIII propondrá la formulación teórica de la separación de poderes que conocemos y aplicamos hoy en día. Para formar un gobierno moderado, nos dice el autor francés, "es necesario combinar los poderes, regularlos, temperarlos, hacerlos actuar, de tal forma que se dé un contrapeso a cada uno de ellos para que puedan resistir la fuerza y la voluntad del otro".(Salazar, 2008)

Esta doctrina se ha vinculado históricamente a la clasificación división tripartita de las funciones estatales. Según esta teoría el poder se divide en tres ramas o funciones: a) la función legislativa que se encarga de la producción de normas generales y abstractas, b) la rama ejecutiva, encargada de ejecutar los mandatos de la ley y del resto de funciones residuales, y, c) la función judicial encargada de la aplicación de las normas en el caso concreto y de vigilar el cumplimiento estricto de las leyes por parte de los otros dos poderes.

Esta garantía institucional, sin embargo, ha sido modificada y relativizada en el derecho contemporáneo, pues hoy en día aparte de que no existen solo tres funciones del Estado ${ }^{\text {ii }}$ cada una de las funciones tradicionales comparte con las otras algunas competencias que en la visión tradicional eran exclusivas de los otros poderes.

Respecto a separación entre Iglesia y Estado, históricamente es un fenómeno que surge durante el Renacimiento y como resultado de las guerras de religión que siguieron a la reforma protestante; se consolida con la ilustración y el racionalismo llegando a ser una política oficial durante la Revolución francesa, la Independencia Norteamericana y las revoluciones burguesas que deshacen la "alianza entre el trono y el altar".

Desde el punto de vista jurídico, cuando hablamos de separación entre iglesia y Estado, nos estamos refiriendo a la garantía jurídica por medio de la cual se consigue que las instituciones públicas y las religiosas se mantengan separadas e independientes mutuamente, Con el resultado de que cada una de ellas mantiene su autonomía para tratar los temas relacionados con sus esferas de influencia. Como resultado de la postivización de tal garantía se logra el establecimiento de un Estado laico, y se inicia el proceso de secularización de una sociedad, mientras en el plano de los derechos esta garantía se manifiesta en el reconocimiento de la libertad de conciencia y de cultos.

En la actualidad, la separación entre la iglesia y el Estado se encuentra plasmada en la mayor parte de las Constituciones de los estados constitucionales modernos En el caso ecuatoriano esta garantía se encuentra reconocida en artículo 1 de la Constitución en virtud del cual el Estado ecuatoriano es entre otros rasgos, Democrático, intercultural, plurinacional y laico. En cuanto atañe a esta última característica los poderes públicos garantizan el laicismo y la ética republicana como sustento del quehacer público y el ordenamiento jurídico.( Mora, 2002)

En cuanto se refiere al principio de legalidad, esta es una de las garantías más importantes del Estado liberal en su versión original, y consiste en la distinción y subordinación de la acción pública y judicial a la ley considerada en sentido formal. Implica como su nombre indica la preeminencia del poder legislativo respecto del resto de poderes y la supremacía de 
la ley respecto del resto de los actos normativos del Estado, incluyendo los derechos fundamentales que solo son tales en virtud de que la ley así lo diga, lo mande y lo permita.

También existe una segunda acepción del principio de legalidad que lo entiende como aquel sometimiento de la administración (ejecutivo) a la ley y se manifiesta en la conocida expresión según la cual "El estado solo puede hacer aquello que está expresamente permitido en la ley mientras que los particulares pueden realizar todo lo que la ley no les prohíbe”.

La principal manifestación de la vigencia del principio de legalidad es justamente el principio de reserva de ley en virtud del cual existen algunas materias que por su importancia social o política el constituyente atribuye exclusivamente al legislador.

En el caso ecuatoriano, por ejemplo, la tipificación de delitos y sanciones, la creación o modificación de impuestos, la regulación de los derechos constitucionales y de cualquier actividad que tenga que ver con el ejercicio de los derechos, la regulación de la organización político administrativa del país, entre otras, solo pueden ser regulados por la ley, o por autorización expresa de la ley.

Ahora bien, con el reconocimiento de la supremacía de la Constitución este principio de legalidad, principio y fin del Estado de derecho decimonónico se relativiza y se convierte en principio de juridicidad o constitucionalidad, en virtud del cual los atributos que antes se predicaban de la ley, (imperatividad, generalidad, coercitividad, aplicación directa e inmediata) pasan a ser propiedad de la Constitución. A partir de ese momento la palabra ley no es sinónimo de ley en sentido formal, sino que hace referencia a todas las normas del ordenamiento jurídico.

En lo que se refiere a la garantía de la existencia del control de constitucionalidad en cabeza de un órgano autónomo llamado tribunal o corte constitucional, que de acuerdo con la doctrina tiene como misión garantizar la supremacía de la constitución y evitar la dictadura de la mayoría a través de controles que hagan presentes y efectivos los derechos de las minorías políticas.

Con independencia de las criticas recurrentes a las cortes constitucionales, debido al real o supuesto déficit democrático que aqueja a justicia constitucional, es innegable que su existencia significa una transformación revolucionaria del aparato institucional del Estado, porque significa garantizar en los hechos el carácter normativo y la supremacía de la Constitución.

Finalmente, aparece una última garantía institucional: la figura del Defensor del Pueblo que ha sido concebida como una institución de protección extrajudicial de los derechos.

Como se sabe la existencia de la Defensoría del Pueblo, está inspirada en el modelo del ombudsman de los países escandinavos y que fue adoptado con importantes modificaciones por la constitución española e su Art. 54 y a través del constitucionalismo español paso a las constituciones latinoamericanas de las últimas dos décadas.

En el caso ecuatoriano la Defensoría del Pueblo hace parte de la nueva función de transparencia y control social, y tiene como funciones principales la promoción, el ejercicio 
y la divulgación de los derechos humanos, que incluyen la emisión de medidas de cumplimiento obligatorio de los derechos humanos; el patrocinio de las acciones constitucionales; la investigación de acciones y omisiones de servidores públicos en relación con los derechos humanos constitucionalizados; y la vigilancia del cumplimiento estricto del derecho a la libertad, que incluye las acciones necesarias para evitar la tortura y los tratos crueles inhumanos y degradantes; así como solicitar a las autoridades las informaciones necesarias para el ejercicio de sus funciones sin que pueda oponérsele reserva alguna.

Es claro que en este contexto el Defensor del pueblo realiza una importante función controladora de la función administrativa en la medida en que ésta puede llegar a vulnerar los derechos humanos, lo que incluye funciones de control y fiscalización sobre las actuaciones tanto de autoridades como de particulares que cumplan funciones públicas.

Las Garantías de Políticas Públicas: La Constitución ecuatoriana de 2008 a través de la figura de las garantías frente a las políticas públicas incorpora uno de los más importantes avances teóricos del nuevo constitucionalismo latinoamericano respecto de las Constituciones europeas. Por primera vez en la historia constitucional del mundo se vincula la existencia de derechos con la operatividad y obligatoriedad de implementar políticas públicas, es decir que se constitucionaliza y normativiza con el más alto rango la vinculación estrecha que en el Estado democrático existe entre Derechos y Política.

En ese contexto, en el Ecuador posterior al 20 de octubre de 2008, la formulación, ejecución y evaluación de políticas y servicios públicos debe orientarse necesariamente a la eficacia de los derechos del buen vivir, con la consecuencia de que si una política pública vulnera un derecho constitucional hay la obligación de cambiar o reformular la política pública, incluyendo la modificación del presupuesto, con la estrecha participación de los afectados.

Este discurso, por supuesto, representa un cambio copernicano respecto de la eficacia y naturaleza de los derechos sociales, y del discurso sobre carácter normativo y obligatorio de todo el ordenamiento constitucional que en el constitucionalismo europeo continental ha sido desconocido por la consideración de la gran mayoría de los derechos sociales como meros principios o criterios subsidiarios de interpretación a través de su consagración como normas de programación final y principios rectores del ordenamiento, mientras no sean desarrollados por el legislador secundario.(Prieto Sachis, 2007)

A diferencia de lo que ocurre en el constitucionalismo social de los países centrales del capitalismo, en el modelo constitucional del Ecuador, versión 2008, los derechos del Sumak Kawsay no solo están reconocidos taxativamente como derechos públicos subjetivos, con todas las garantías que ello implica, y en tanto tales como facultades jurídicas que hacen parte del patrimonio de las personas, sino que están definidos en el llamado régimen del buen vivir como obligaciones directas del Estado, sancionables a través de las garantías establecidas en el artículo 85 constitucional.

Este conjunto de garantías extrajudiciales se pueden entender como garantías construidas en función del modelo de separación de poderes, mismo que esquemáticamente puede ser representado a través de la siguiente matriz: 
Tabla 1. Análisis de las garantías según los poderes del Estado

Garantías en función de la separación de poderes

\begin{tabular}{|c|c|c|c|c|}
\hline $\begin{array}{l}\text { Función } \\
\text { legislativa }\end{array}$ & $\begin{array}{l}\text { Función } \\
\text { Ejecutiva }\end{array}$ & $\begin{array}{l}\text { Función } \\
\text { judicial }\end{array}$ & $\begin{array}{l}\text { Función de } \\
\text { Control } \\
\text { Constitucional, }\end{array}$ & $\begin{array}{l}\text { función de } \\
\text { transparencia y } \\
\text { Control social }\end{array}$ \\
\hline Garantías & $\begin{array}{l}\text { Garantías } \\
\text { de }\end{array}$ & Garantías & \multirow{4}{*}{$\begin{array}{l}\text { Garantías } \\
\text { Institucionales }\end{array}$} & \multirow{4}{*}{$\begin{array}{l}\text { Garantías } \\
\text { institucionales }\end{array}$} \\
\hline Normativas & Políticas & Jurisdiccionales & & \\
\hline Art. & públicas & Art. 86 a 84 & & \\
\hline CRE & $\begin{array}{ll}\text { Art. } & 85 \\
\text { CRE } & \end{array}$ & $\mathrm{CRE}$ & & \\
\hline
\end{tabular}

Fuente: Constitución de la República del 2008

Las Garantías jurisdiccionales en las democracias constitucionales contemporáneas la forma de garantizar derechos más conocida es aquella que se ejerce ante la jurisdicción, comúnmente conocida como garantías jurisdiccionales de los derechos. Estas no son otra cosa que la posibilidad de ejercer el derecho de acción para lograr la tutela efectiva de los derechos. Y en cuanto tales son un Conjunto de instrumentos procesales que -dentro del sistema jurídico estatal- cumplen la función de la tutela directa de los derechos Constitucionales.

Esto tiene que ver igualmente con el nuevo papel que los jueces cumplen en las democracias constitucionales modernas. En efecto, la necesidad de garantizar institucionalmente los derechos humanos y la crisis del parlamentarismo racionalizado han producido un redimensionamiento de la función jurisdiccional dentro de la estructura del Estado: el juez ya no es más como pensaba Montesquieu, y los liberales del siglo XIX, la boca muda de la ley sino que se convierte en el protagonista de la acción del Estado, a través de su papel de garantes últimos de los derechos fundamentales, y con ellos las garantías procesales se convierten en un elemento esencial de este nuevo modelo.

Esta mutación en la concepción de la jurisdicción no ha sido, sin embargo un proceso pacífico: Los poderes públicos tradicionales, afectados por esta nueva cultura jurídica, ven con profunda desconfianza el nuevo rol de los jueces y han comenzado a plantear la necesidad de realizar reformas -que limiten la autonomía del poder judicial y restauren el antiguo equilibro entre poderes propio del liberalismo decimonónico.

Las principales garantías judiciales conocidas en los ordenamientos jurídicos occidentales son: el habeas corpus, la acción de amparo, tutela o protección, el habeas data, las acciones de grupo y el amparo contra sentencias judiciales. En el caso ecuatoriano la Constitución de 1998 fue la primera que reconoció en el plano constitucional este tipo de amparos 
jurisdiccionales, pero lo hizo de manera desordenada y asistemática, pues tan solo reconoció la existencia del amparo, el habeas corpus y el habeas data.

La Constitución de 2008 en ese sentido, representa un avance sustancial frente a la anterior constitución pues establece un amplio y completo abanico de garantías jurisdiccionales organizadas, como se verá en el cuadro que aparece a continuación, en función de los derechos protegidos.

Tabla 2: Análisis comparativo de las garantías entre la Constituciones 1998 y 2008

\begin{tabular}{|c|c|c|}
\hline Derecho Protegido & Constitución de 1998 & Constitución de 2008 \\
\hline \multirow{2}{*}{ Libertad y derechos Conexos } & Habeas Corpus & Habeas Corpus \\
\hline & Administrativo & Judicial \\
\hline $\begin{array}{l}\text { Resto Derechos } \\
\text { fundamentales }\end{array}$ & $\begin{array}{l}\text { Amparo preventivo y } \\
\text { Reparatorio }\end{array}$ & Acción de Protección \\
\hline $\begin{array}{l}\text { Transparencia y Acceso a la } \\
\text { información publica }\end{array}$ & $\begin{array}{l}\text { Amparo legal no } \\
\text { constitucional }\end{array}$ & $\begin{array}{l}\text { Acción de acceso a la información } \\
\text { pública }\end{array}$ \\
\hline Datos personales & Habeas Data judicial & Habeas Data judicial \\
\hline Debido proceso Judicial & $\begin{array}{l}\text { No hay Garantía judicial ni } \\
\text { administrativa }\end{array}$ & $\begin{array}{l}\text { Acción Extraordinaria de } \\
\text { Protección }\end{array}$ \\
\hline
\end{tabular}

Fuente: Constituciones de 1998 y 2008

De acuerdo con el Art. 6 Ley Orgánica de Garantías Jurisdiccionales y Control Constitucional las garantías jurisdiccionales buscan la protección eficaz e inmediata de los derechos reconocidos en la Constitución y en los instrumentos internacionales de derechos humanos, la declaración de la violación de uno o varios derechos, así como la reparación integral de los daños causados por su violación.

\section{Resultados}

Del estudio normativo, conceptual y doctrinario de los preceptos constitucionales y legales en el Ecuador, hemos obtenido como resultado, que vislumbramos la configuración de un sistema armónico y entrelazado con cuatro tipos de garantías de protección de derechos, que son: Garantías institucionales, Garantías Normativas, Garantías de Políticas Públicas y Garantías Jurisdiccionales. Cada una de aquellas tienen su propia peculiaridad y naturaleza, pero se encuentra vinculada en mayor o menor medida a las otras de tal manera que se puede señalar que tenemos un sistema armónico e integral, que complementa la una con la otra en determinados momentos y que constituye a mi entender un sistema integral de protección de derechos fundamentales. Las Garantías institucionales están vinculadas a una serie de preceptos, principios e instituciones que garantizan derechos. Las garantías normativas, establecen la imposibilidad de que el órgano público con potestad legislativa emita normas generales que vulneren derechos. La garantías de políticas públicas, a la acción del poder ejecutivo y los gobiernos autónomos descentralizados en el ámbito de sus competencia, respecto a planes y programas a desarrollare para hacer efectivos los derechos; y finalmente 
la garantías jurisdiccionales, que dan a la función judicial la potestad de hacer cumplir y respetar los derechos fundamentales

\section{Conclusiones}

- La normativa constitucional ecuatoriana, en lo que corresponde a su parte dogmática, es decir a la que se establece un amplio catálogo de derechos y un conjunto de garantías, se nutrió de un contenido teórico doctrinal importante, de los más avanzados en materia de garantía de derechos; así como recogió estándares del Derecho Internacional de los Derechos Humanos.

- Del estudio detallado de este grupo de garantías, he llegado a determinar que entre ellas existen relaciones imperceptibles que nos dan algunos elementos para teorizar ejes sistémicos en la protección de los derechos fundamentales en Ecuador, mas no una simple agrupamiento aislado como normal y comúnmente se entiende. Por lo anterior, se recomienda a) Continuar estudiando desde la perspectiva teórica y de la praxis institucional la aplicación sistémica de la garantía de los derechos fundamentales.; b) Difundir la sistematización de los mecanismos de protección en nuestro país, para su mejor entendimiento y aplicación, cumpliendo de esa manera el postulado de que la mayor obligación del Estado y su primer deber el cumplir y hacer cumplir los derechos fundamentales.

\section{Referencias bibliográficas}

De Padua, M . (2001), Defensor Pacis, USA, Columbia University Press.

Hobbes, TH. (1980), El Leviatán, México, FCE.

Locke J. (2002), Segundo Ensayo sobre el Gobierno Civil, Buenos Aires, Editorial Lozada.

Rousseau JJ. (1983), El Contrato Social, Madrid, Editorial Sarpe.

Guastini R.(2009), La Constitucionalización del Ordenamiento Jurídico, En: Carbonell M. (Ed) Neoconstitucionalismos, Madrid, Editorial Trota.

Pérez Luño, A. (1998), Los Derechos Fundamentales, Madrid, Editorial Tecnos

Villa Casado I.(2007) Fundamentos del Derecho Constitucional Contemporáneo, Bogotá, Editorial Legis

Montesquieu, Ch,(1971), El Espíritu de las leyes, México, Editorial Porrúa.

Salazar Ugarte P.(2008), La democracia Constitucional, México, FCE.

Mora, Luis. (2002), Jurisdicción Constitucional en América Latina, Quito, Corporación Editora Nacional.

Prieto Sanchis, L.(2007), Teoría del Derecho, Madrid, Editorial Trota. 


\section{PARA CITAR EL ARTÍCULO INDEXADO.}

Mancero Carrillo, P. (2019). Teorización sistémica de las garantías de los derechos fundamentales en Ecuador. Ciencia Digital, 3(3.2.1), 228-238. https://doi.org/10.33262/cienciadigital.v3i3.2.1.815

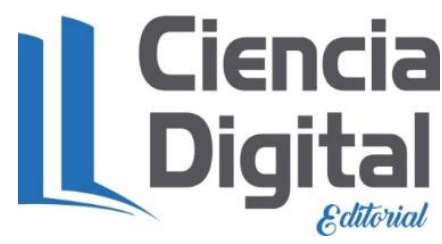

El artículo que se publica es de exclusiva responsabilidad de los autores y no necesariamente reflejan el pensamiento de la Revista Ciencia Digital.

El artículo queda en propiedad de la revista y, por tanto, su publicación parcial y/o total en otro medio tiene que ser autorizado por el director de la Revista Ciencia Digital.
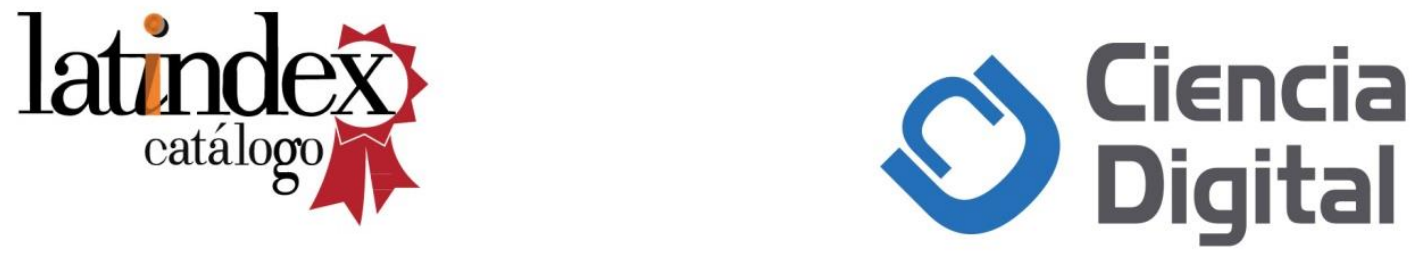